\title{
Prevalence of Hyper Amylasemia and Acute Pancreatitis in Organophosphate Poisonings
}

\author{
Dr. Abhay Nath Chaturvedi ${ }^{1}$, Dr. Soumyakanti Dutta ${ }^{2}$, Dr. Sanjay Sarkar ${ }^{3}$, \\ Dr. Tushar Kanti Saha ${ }^{4}$, Dr. Subhajyoti Adhikary ${ }^{1}$, Dr. Sukdeb Das ${ }^{5}$, \\ Dr. Mitali Basu ${ }^{6}$ \\ $\left\{{ }^{1}(P G T),{ }^{3}\right.$ (RMO), ${ }^{5}$ (Asso. Prof.), ${ }^{6}$ (Asst. Prof.) $\}$ Dept. of Medicine, Bankura Sammilani Medical College \& \\ Hospital, Bankura; ${ }^{2}$ (Senior Resident, Dept. of Cardiology, Bankura Sammilani Medical College \& Hospital, \\ Bankura); ${ }^{4}$ (Asst. Prof., Dept. of Community Medicine, NRS Medical College, Kolkata)
}

\begin{abstract}
Objective: To determine the frequency of hyperamylasemia and acute pancreatitis following organophosphate poisoning. Methodology: This is a cross sectional study conducted at the Medicine Department, B.S. Medical College, West Bengal during the period of six months from 16th June 2012 to 15th June 20013. All patients of both sexes and ages above 12 years admitted with a positive history of organophosphate poisoning $(O P)$ were included in the study. A special Proforma was designed to enter all the collected data containing the basic information about the patient, history of recent event and the past history, physical examination and the relevant investigations like complete blood count, serum amylase and lipase, alanine aminotransferase (ALT), lactate dehydrogenase (LDH),ultrasound abdomen CT abdomen(where needed). Result: Among 96 patients, hyperamylasemia was found in 30 (31.25\%) patients. Acute pancreatitis was seen in $6(6.25 \%)$ patients. Conclusion: Hyperamylasemia is more frequently seen in organophosphate poisoning while six patients proved to have acute pancreatitis as a complication.
\end{abstract}

Keywords: Organophosphate Poisoning, Acute Pancreatitis, Hyperamylasemia.

\section{Introduction}

The incidence of organophosphate (OP) poisoning is sobering and it can occur in several settings. Worldwide there were approximately 3 million organophosphate poisonings last year and approximately 200,000 people died. One-fifth of the cases were occupational and the mortality rate was slightly less than one percent. Self-inflicted poisonings were responsible for over ninety percent of the exposures worldwide with a mean age of twenty- five years and a mortality rate between four to thirty percent among this group of exposures. Organophosphorus insecticides include chlorpyrifos, phosphorotioic acid (Diazinon), dichlorvos, fenthion, parathion and malathion, and carbamate insecticides include aldicarb, propoxur, carbaryl and bendiocarb. The nerve gas, sarin, is also an OP. OP insecticides irreversibly inhibit acetylcholine esterase and cause accumulation of acetylcholine at muscarinic and nicotinic synapses and in the CNS. Cholinergic stimulation of the pancreas and the Sphincter of Oddi (SO) results in both increased pancreatic secretion and increased SO activity in animal models. It has been shown that excessive cholinergic stimulation using an acetylcholine agonist can result in acute pancreatitis. Organophosphate used as an insecticide irreversibly inhibits cholinesterase resulting in delayed breakdown of synaptic acetylcholine and has been noted to cause acute pancreatitis in humans. In animal models organophosphates results in acute pancreatitis associated with raised pancreatic duct pressure. This is thought to be secondary to "obstruction" at the SO level coupled with cholinergic stimulation of pancreatic secretion. ${ }^{1}$ This study was therefore designed to determine the frequency of hyperamylasemia and acute pancreatitis in organophosphate poisoning in our setup and to reduce mortality and hospital stay by its early detection.

\section{Methodology}

This is a cross sectional hospital based study carried out in B.S.Medical College, Bankura,West Bengal which is tertiary care facility catering for acute and sub-acute emergencies. The study was conducted over a period of one year from June 16th 2012 to June 15th 2013.

A total of 96 patients ( 65 females and 31 males) of acute poisoning with organophosphate insecticide were studied. All patients including both sexes and ages above 12 years with a recent history of organophosphate ingestion, inhalation or cutaneous absorption with clinical sign and symptoms of organophosphate poisoning were included in the study. Confirmation of the poison was done by seeing the container bought by the patient's family members and composition printed over them.

Those patients with history of ingestion of any other material along with organophosphates, who were addicted to alcohol or had a history of gallstones or gallstones seen on ultrasound or any other history of gastric 
ulcer or burning epigastric pain in past were excluded from the study. Also those who had gone through ERCP (endoscopic retrograde cholangiopancreatography) in previous 24 hours or had a positive history of drug intake like azathioprine, mercaptopurine, asparginase, valproic acid, pentamidine, estrogens etc. were not included in the study.

Serum amylase and lipase levels were measured with a Hitachi-911 autoanalyser via enzymatic and colorimetric assay. Serum amylase levels between 0 and $96 \mathrm{U} / \mathrm{L}$ and serum lipase levels between 0 and 60U/L were accepted as normal. Any two of the following criteria were used to diagnose acute pancreatitis: typical abdominal pain, threefold or greater elevation in serum amylase and/or lipase level, and/or confirmatory findings on cross-sectional abdominal imaging ${ }^{2}$. All patients diagnosed to have acute pancreatitis also underwent an ultrasonographic study (by experienced radiologists) and computerized tomography, if necessary. Also the serum levels of SGPT, LDH, CPK and leukocytes counts were measured.

Data Collection Procedure: A special Proforma was designed to enter all the collected data containing the basic information about the patient, history of recent event and the past history, physical examination and lab investigations including complete blood count, random blood sugar, alanine aminotransferase (ALT), lactate dehydrogenase, serum amylase and lipase and ultrasound abdomen.

Data Analysis: The collected data was analyzed statically using SPSS version-10 on computer. Descriptive statistics like frequency and percentage of qualitative variables like sex, history, presenting complaints, clinical findings and causes of pancreatitis including gallstones, alcohol addiction, drug intake etc and quantitative variables like age, weight, amount of pesticide ingestion, time of ingestion and stomach wash done were presented by mean and standard deviation.

\section{Result}

During period of study 96 patients with ingestion of organophosphates were evaluated. 80 among 96 patients intended to commit suicide $(83.33 \%)$, whereas 16 patients, $(16.67 \%)$ were exposed to organophosphates due to accidental event. None came with a homicidal attempt.

92 patients $(95.83 \%)$ were exposed to organophosphates through gastrointestinal route, two patients via inhalation (2.08\%), and two by cutaneous absorption $(2.08 \%)$. Among 96 patients sex distribution showed 65 patients $(67.70 \%)$ were females and 31 patients $(32.29 \%)$ were males. Male to female ratio was $0.48: 1$. Among 65 females $55(84.6 \%)$ had intended suicidal attempt. 10 females (15.38\%) gave statement of accidental intake of organophosphates. Among 31 males $25(80.65 \%)$ males were exposed to organophosphates as a suicidal attempt and $6(19.35 \%)$ were accidental. Mean age at presentation was 25 years. The youngest was 12 years old whereas the oldest was 72 years old.

Through the one year period 30 of 96 patients $(31.25 \%)$ were found to have hyperamylasemia. 16 patients $(16.67 \%)$ had more than threefold elevated amylase levels. Total 13 patients (10\%) out of 30 with hyperamylasemia, had raised lipase levels (>60 IU), among them seven had less than threefold elevation of lipase while 6 patients had three fold elevation of lipase. Among 96 patients 30 with hyperamylasemia invariably had raised leucocyte count, raised SGPT, LDH and CPK (Table-I).

Table - 1: Showing changes in some biochemical parameters according to serum amylase levels

\begin{tabular}{|l|l|l|l|l|}
\hline & $\begin{array}{l}\text { Serum amylase } \\
\text { level( } \leq 96 \mathrm{U} / \mathrm{L}) \\
(\text { No. of patients) }\end{array}$ & $\begin{array}{l}\text { Serum amylase level(97- } \\
\text { 288 U/L) } \\
\text { (No. of patients) }\end{array}$ & $\begin{array}{l}\text { Serum amylase level( } \\
>288 \text { U/L) } \\
\text { (No. of patients) }\end{array}$ & Total(\%) \\
\hline $\begin{array}{l}\text { Leucocyte } \\
\text { count }\left(>10 \times 10^{3} / \mathrm{mm}^{3}\right)\end{array}$ & $13(13.54 \%)$ & $14(14.58 \%)$ & $16(16.67 \%)$ & $44.79 \%$ \\
\hline SGPT( $>41 \mathrm{U} / \mathrm{L})$ & $2(2.08 \%)$ & $13(13.54 \%)$ & $15(15.62 \%)$ & $31.25 \%$ \\
\hline $\mathrm{LDH}(>450 \mathrm{U} / \mathrm{L})$ & $6(6.25 \%)$ & $12(12.5 \%)$ & $14(14.58 \%)$ & $33.33 \%$ \\
\hline $\mathrm{CPK}(>200 \mathrm{U} / \mathrm{L})$ & $3(3.12 \%)$ & $10(10.42 \%)$ & $12(12.5 \%)$ & $26.04 \%$ \\
\hline
\end{tabular}

Among the patients with normal amylase levels, isolated elevated leucocyte count was noted in 13 patients (13.54\%), isolated raised LDH was found in 6 patients $(6.25 \%)$, raised CPK seen in 3(3.12\%) patients and raised SGPT was seen in two patients (2.08\%). Among 96 patients the most common clinical presentation was excessive salivation seen in $45(46.87 \%)$ of patients. Retching only was observed in 31patients $(32.29 \%)$ while vomiting was seen in $23(23.96 \%)$ of patients. Abdominal pain was seen in 25 patients $(26.04 \%)$, out of these 15 had hyperamylasemia and 6 had acute pancreatitis proven by raised lipase levels and ultrasound findings. Confusion and drowsiness was noted in 22 patients (22.92\%).

The frequently observed clinical findings among 96 patients with a positive history of ingestion of organophosphates were constricted pupils seen in 60 patients (62.5\%). Fasciculations were noted in 34 patients $(35.41 \%)$. Chest crepitations were heard in $16(16.67 \%)$. Respiratory depression was observed in 10 patients $(10.45 \%)$. Convulsions were seen in $4(4.17 \%)$. Among 96 patients $26(27.08 \%)$ have no significant clinical 
findings on presentation. 5 patients died during the study, 4 due to respiratory depression and one due to convulsions and aspiration.

\section{Discussion}

Cases with acute pancreatitis as a complication of organophosphate exposure have been reported in the literature ${ }^{3}$. Ahmed Arshia et al $^{4}$ conducted a descriptive study at the Medicine Department, Abbasi Shaheed Hospital Karachi during the period of six months from 16th June 2006 to December 2006. All patients of both sexes and ages above 15 years admitted with a positive history of organophosphate poisoning (OP) were included in the study. Among 90 patients, hyperamylasemia was found in $28(31 \%)$ patients. Hyperlipasemia was seen in nine (10\%) patients and pancreatitis was seen in two (2.2\%) patients. Concluded that hyperamylasemia is more frequently seen in organophosphate poisoning while two patients proved to have acute pancreatitis as a complication.

S Singh et $\mathrm{al}^{5}$ carried out a prospective study in PGIMER, Chandigarh, India between June 2001-June 2005, to find the incidence of hyperamylasemia and acute pancreatitis in patients with OP poisoning. Of the 79 patients studied, serum Amylase was found to be elevated (> 200 S.U) in 37 patients (46.95\%), among them in three patients it was $800 \mathrm{~S}$.U. One of them showed swollen pancreas on ultrasonography which was confirmed by Computerised Tomography. In other two patients, evidence of pancreatitis was not observed. There was no significant correlation between the nature of compounds (OP or carbamates), duration and severity of cholinergic syndrome and increase in serum Amylase. It has been concluded that mild elevation of serum Amylase is common in patients with OP poisoning, however acute pancreatitis is rare.

I Sahin et $\mathrm{al}^{6}$ conducted a prospective study in the Department of Internal Medicine, University of Yuzuncu Yil, Medical faculty,Van,Turkey in 2002, to find the prevalence of pancreatitis in OP poisoning. Four of the total 47 patients with acute OP poisoning had obviously elevated Amylase and Lipase levels (Amylase> $300 \mathrm{U} / \mathrm{L}$; Lipase $>60 \mathrm{U} / \mathrm{L}$ ). Only two of the patients with Amylase levels between 100 and $300 \mathrm{U} / \mathrm{L}$ had elevated levels of Lipase. None of the patients with normal Amylase levels had elevated levels of Lipase. A total of $12.76 \%$ were diagnosed as acute Pancreatitis. It was concluded that acute pancreatitis is not a rare complication of organophosphorus poisoning. In order to improve the outcome of OP poisoning early diagnosis of acute pancreatitis is important and serum Amylase and Lipase levels should be routinely considered carefully.

W C Lee et $\mathrm{al}^{7}$ carried out a retrospective study of medical records of 121 patients with the diagnosis of OP poisoning over three years in Veterans General Hospital, National Yang- Ming University in 1998. Serum amylase, pancreatic amylase, salivary amylase, lipase and cholinesterase levels and the clinical manifestations were analyzed. It was observed that 44 patients $(36 \%)$ had hyperamylasemia (Amylase $>360 \mathrm{U} / \mathrm{L}$ ). Lipase was measured in 28 patients with hyperamylasemia; nine of 28 had hyperlipasemia (Lipase > $380 \mathrm{U} / \mathrm{L}$ ). The finding of hyperamylasemia was closely related to clinical severity and presence of shock. It was concluded that hyperamylasemia is frequent in severe OP poisoning. However, hyperamylasemiais not synonymous with acute pancreatitis and pancreatic amylase is not reliable parameter in the diagnosis of organophosphate induced pancreatitis due to its low sensitivity and specificity. Lipase assay is indicated in patients with hyperamylasemia for early diagnosis of pancreatitis.

$\mathrm{N}$ Matsumiya et $\mathrm{al}^{\mathbf{8}}$ performed a retrospective study of OP poison in intensive care unit to analyze the incidence of respiratory failure in Department of Anesthesiology \& Critical care medicine, Kyodo General Hospital, Ibaraki, Japan in 1996. Of the 32 OP poisoning patients, 16 developed respiratory failure and received ventilator support. An increase in plasma amylase above the normal range was found in patients who developed respiratory failure. Thus in $\mathrm{OP}$ poisoning, the elevation of amylase levels was predictive of subsequent respiratory failure.

However in our study, we observed frequent hyperamylasemia in $31.25 \%$ which is slightly lower as observed in other studies. In this study the percentage of pancreatitis in our setup is lower as compared to the studies conducted internationally but higher in comparison with Ahmed Arshia et al and Singh et al. We found that elevation of serum amylase is common in patients with pesticide poisoning. However, acute pancreatitis is also not uncommon.

We also observed that those 6 patients with acute pancreatitis had a delayed transportation to hospital. Time lapse between ingestion of insecticide and reaching to hospital and subsequent management was more than four hours as compared to those who had arrived to hospital within two hours. We have also observed elevation in the serum levels of SGPT, LDH, CPK and TLC in all patients showing raised amylase and lipase.

Limitation of our study was that serum cholinesterase levels were not done on admission because of non availability of test in our hospital lab. 


\section{Conclusion}

Acute pancreatitis is not a rare condition in OP poisonings. Diagnosis of acute pancreatitis should be given importance and appropriate treatment can be life-saving, there should be more studies focused on this aspect of organophosphate poisoning.

\section{References}

[1] Sphincter of Oddi dysfunction and acute pancreatitis - CHEN et al. 43 (3 http://gut.bmj.com/content/43/3/305.full

[2] Greenberger NJ et al,acute and chronic pancreatitis, Hrrison's textbook of internal medicine $18^{\text {th }}$ edition,chapter $313,2637$.

[3] Greenberger NJ, Toskas PP, Isselbacher KJ. Acute and chronic pancreatitis. In Fauci SA, Braunwald E, Isselbacher KJ, et al eds. Harrison's principles of internal medicine, 14th edition. New York: McGraw- Hill, 1998: 1741-52

[4] Ahmed A, Begum I, Aquil N, Atif S, Hussain T, Vohra EA. Hyperamylasemia and acute pancreatitis following organophosphate poisoning. Pak J Med Sci 2009;25 (6):957-961

[5] Singh S, Bhardwaj U, Verma SK, Bhalla A, Gill K..Hyperamylasemia and acute pancreatitis following anticholinesterase poisoning. Hum ExpToxicol 2007 Jun;26(6):467-71

[6] Sahin I, Onbasi K, Sahin H, Karakaya C, Ustun Y, Noyan T. The prevalence of pancreatitis in organophosphate poisonings. Hum Exp Toxicol. 2002 Apr;21(4):175-7

[7] Wui-Chiang Lee, Chen-Chang Yang, Jou-Fang Deng, Ming-Ling Wu, JiinGer, Han-Chieh Lin, Full-Young Chang and Shou-Dong Lee. The Clinical Significance of Hyperamylasemia in Organophosphate Poisoning. J Toxicol Clin Toxicol 1998, Vol. 36, No.7 , Pages 673-681

[8] Matsumiya N, Tanaka M, Iwai M, Kondo T, Takahashi S, Sato S. Elevated amylase is related to the development of respiratory failure in organophosphate poisoning. Hum Exp Toxicology.1996 Mar;15(3):250-3 